\title{
Research Paper: Comparison of Participation of Children With Cerebral Palsy Aged 4 to 6 years in Occupations With Normal Peers
}

\author{
Omid Rostam Zadeh ${ }^{1},{ }^{*}$ Malek Amini ${ }^{1}$, Afsoon Hasani Mehraban ${ }^{1,2}$
}

1. Department of Occupational Therapy, School of Rehabilitation Sciences, Iran University of Medical Sciences, Tehran, Iran. 2. Rehabilitation Research Center, School of Rehabilitation Sciences, Iran University of Medical Sciences, Tehran, Iran.

Citation: Rostam Zadeh O, Amini M, Hasani Mehraban A. [Comparison of Participation of Children With Cerebral Palsy Aged 4 to 6 in Occupations With Normal Peers (Persian)]. Archives of Rehabilitation. 2016; 17(3):192-199. http://dx.doi.org/10.21859/jrehab-1703252

: http://dx.doi.org/10.21859/jrehab-1703192

Received: 01 Feb. 2016

Accepted: 14 May 2016

Keywords:

Cerebral palsy,

Child, Occupation,

Participation

\section{ABSTRACT}

Objective The participation of children in occupations, such as self-care, home environment, play, leisure, and educational and social environments, is significantly correlated with their health and wellbeing. Therefore, the aim of this study was to compare the children with cerebral palsy (CP) and their normallydeveloping counterparts in terms of their participation in each area of occupations.

Materials \& Methods This case-control study was conducted in 2015 with 50 children (aged 4-6 years) having CP and 50 normally-developing children (aged 4-6 years) in the Tehran city. The participants were chosen by a convenience sampling strategy and examined using the Persian version of Children Participation Questionnaire. The questionnaire was completed by the parents after the necessary explanations. SPSS (version 18) was used for statistical analysis, and independent t-test was used for the comparison. Results The mean ages of children with CP and normally-developing children were 5 years and 5.1 years, respectively. Significant differences $(\mathrm{P}<0.001)$ were found in diversity, intensity, enjoyment, assistance, and parent satisfaction with participation in the areas of self-care, home environment, play, leisure, education of children with CP and their peers. However, there was no significant difference in the area of social participation between the two groups $(P>0.05)$.

Conclusion The results showed that the children with $\mathrm{CP}$ have a lower level of participation in occupations compared to their normally-developing counterparts. One of the reasons for the low participation level of children with CP could be the lack of goal-oriented and occupation-oriented interventions. Thus, this finding will help the healthcare providers to create and develop appropriate therapeutic and rehabilitation interventions so as to meet the needs of these children and promote their participation level and wellbeing. 


\title{
مقايسه مشاركت كودكان فلج مغزى f تا وسال با كودكان همسال طبيعى در كارهها
}

\author{
اميد رستمزاده'، "مالك امينى'، افسون حسنىمهربان 'وr'

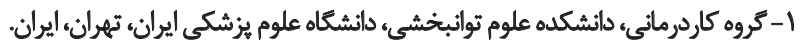

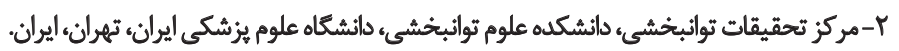

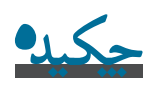

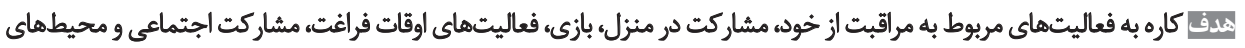

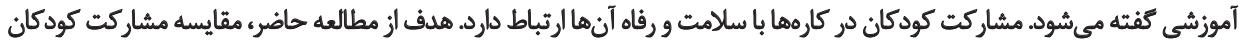

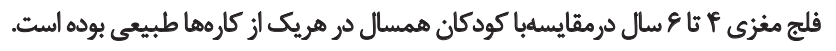

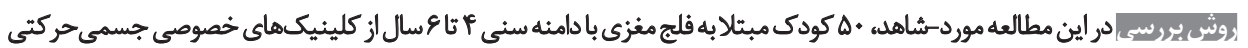

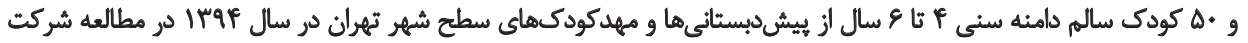

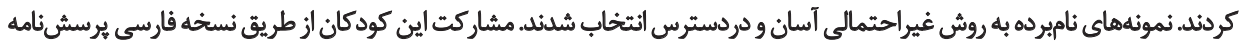

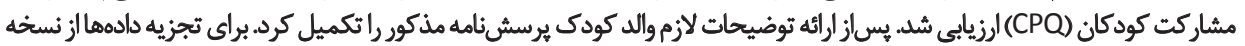

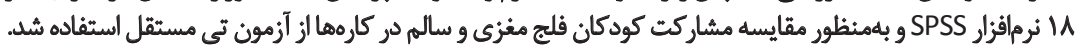

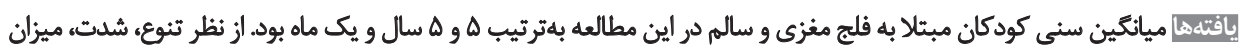

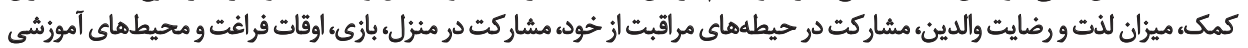

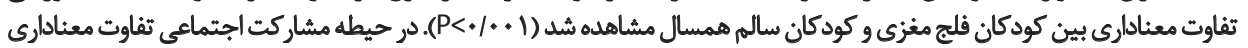

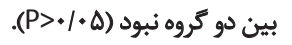

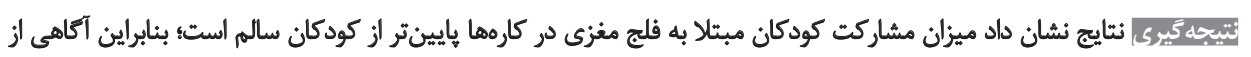

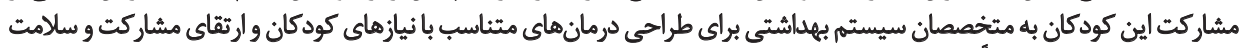

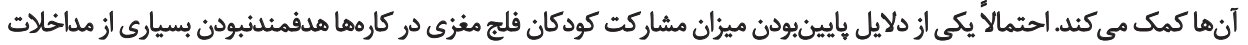
و استفادمنكردن از كارهها در مداخلات استيت.

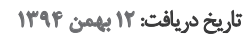

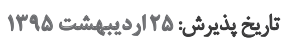

در معرض خطر كاهش مشاركت بـ فر فعاليتهاى اوقات فراغت نيز

مقدمه

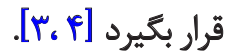

مشاركت در كارههاى روزمره دوران كودكى براى رشد و تكامل

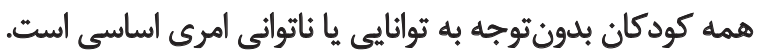

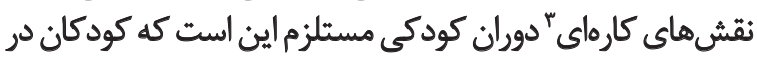

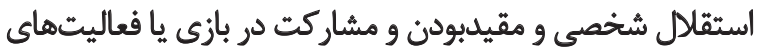

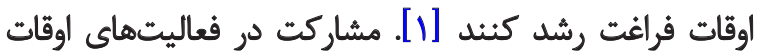

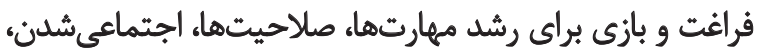
كشف علايق شخصى و زندكي لذتبخش مازي لازم است [ب]. نقص

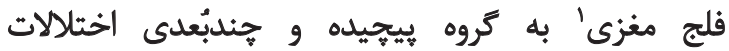

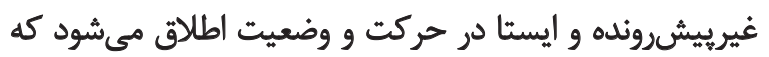

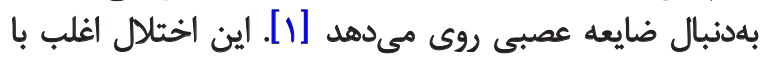

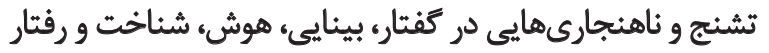

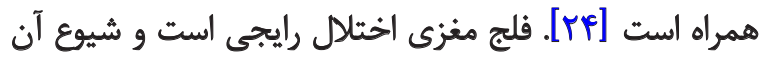

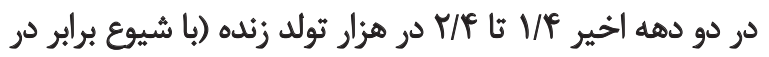

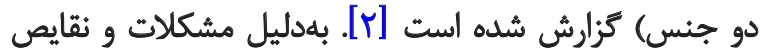

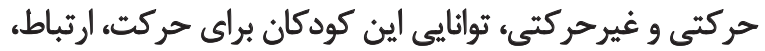

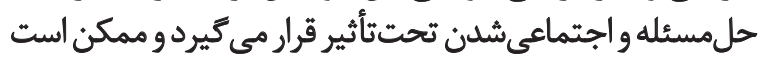

2. Participation

3. Occupational

1. Cerebral palsy

- 
و جامعه، اجتماعى و زندگى مدنى (WHO) [IT]

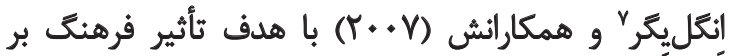

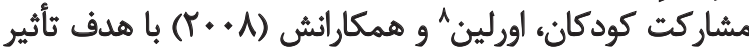

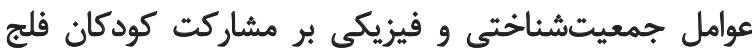

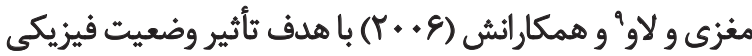

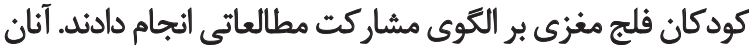

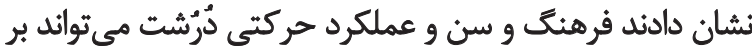

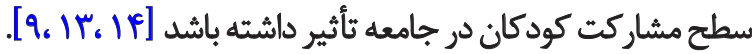
در ايران نيز مطالعاتى درزمينه مشاركت كودكان فلج مغزى انجام

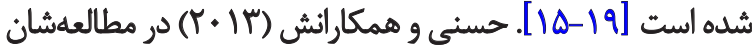

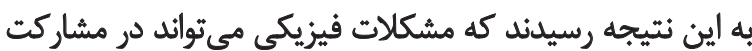

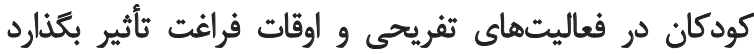

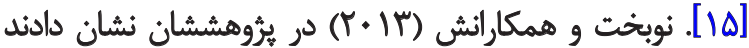
كه برطرفكردن موانع محيطى و افزايش تسهيل ونيل كنيندهها ممكن

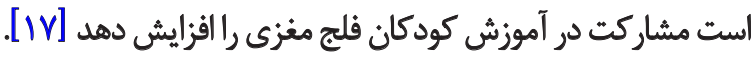
باوجوداين براساس جستجوى انجامشده ثاكنون مطالعهاي در

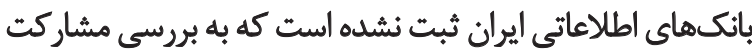

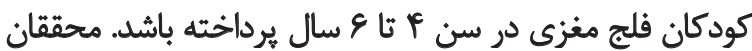

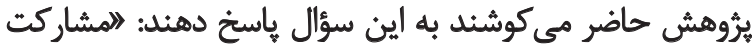

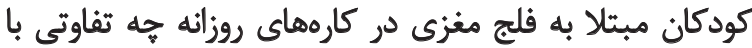

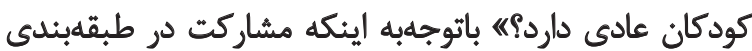

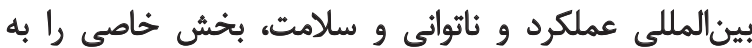

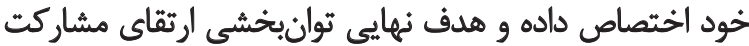

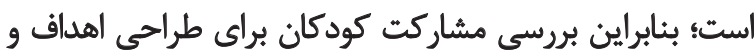

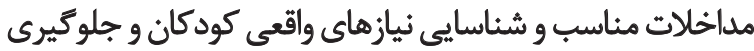

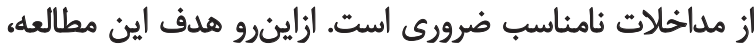
مقايسه مشاركت كودكان فلج مغزي fi أنا

$$
\text { كودكان همسال طبيعى است. }
$$

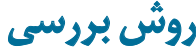

اين مطالعه از نوع موردشاهاهد بود كه در آن مشاركت در

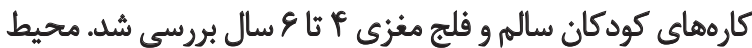

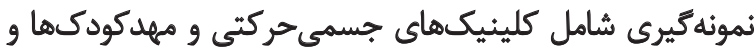

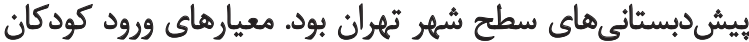

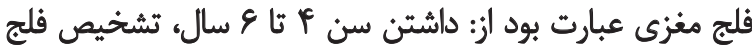

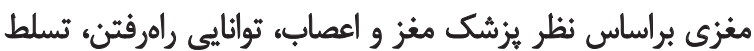

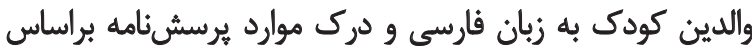

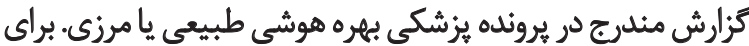

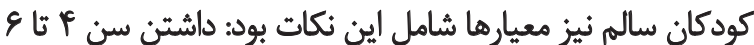

در مشاركت در انجام كارهاى روزمره مي توائد منجر به انزوا و

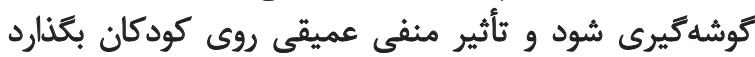

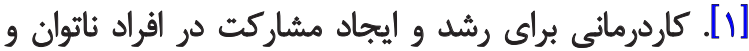

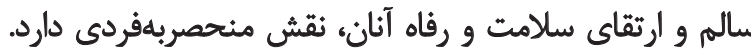

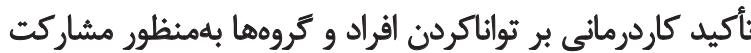

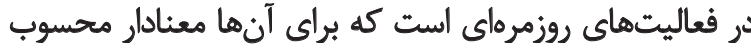

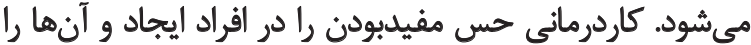
براى زندكى روزانه با ديكران آماده مى كند [هادئ.

طبقهبندى بينالمللى عملكرد و ناتوانى و سلامت (ICF)"

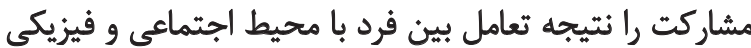

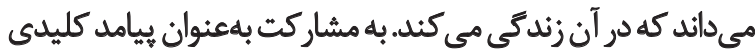

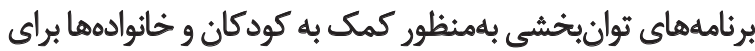

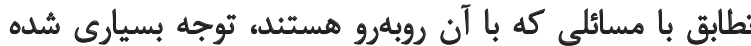

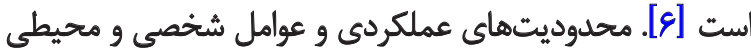

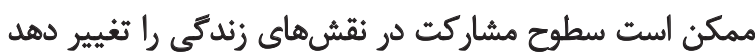

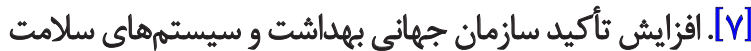

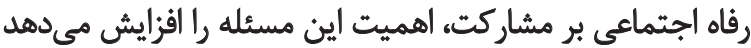

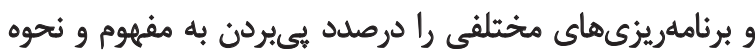

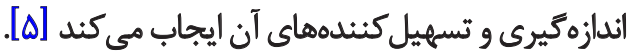

براساس تعريف سازمان بهداشت جهانى (WHO)؛، مشاركت

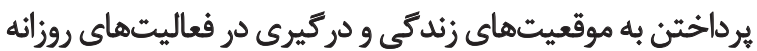

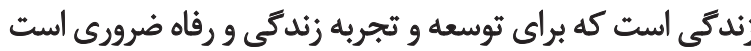

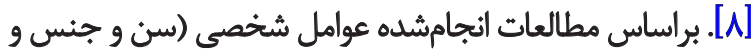

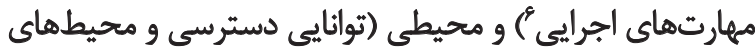
اجتماعىاقتصادى، فرهنكيى و و حمايتى) بر الكوى (توائى مشاركت

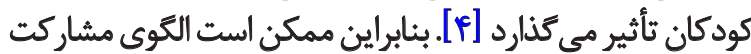

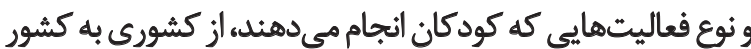

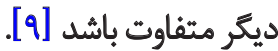

درحال حاضر مشاركت بهعنوان محصول نهايى توانبخشي،

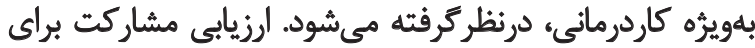

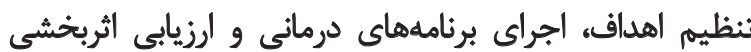

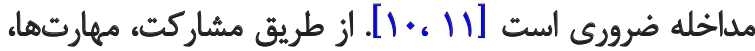

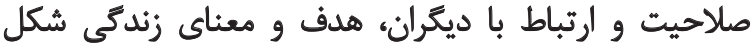

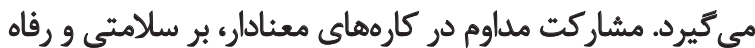

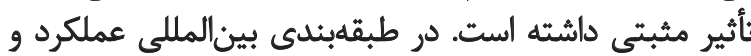

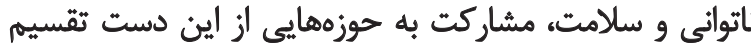

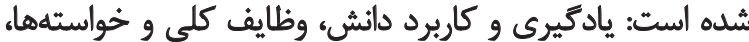

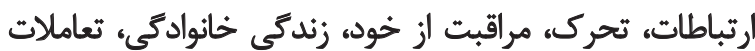

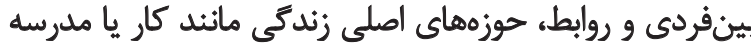

4. International Classification of Functioning, Disability \& Health (ICF) 5. World Health Organization (WHO)

6. Performance skills 
نمره هى دهند: ا. تعلداد دفعات مشاركت (نمره بالا ه و ونمره يايين

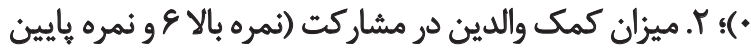

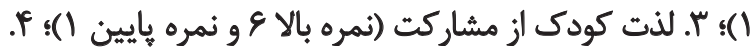

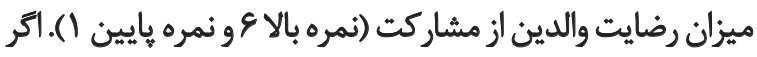

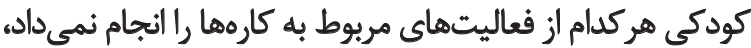

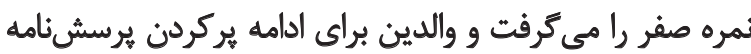

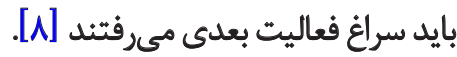

در اين مطالعه براى تجزيه دادهها از نسخه

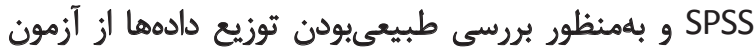

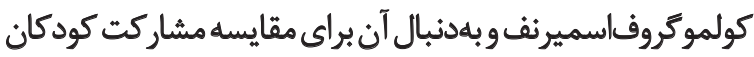

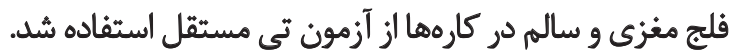

يافتهها

نتايج سنجش طبيعىبودن دادهها نشان داد كه توزيع دادهها

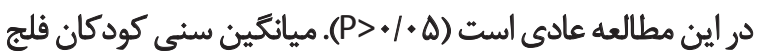

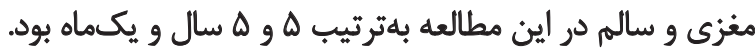

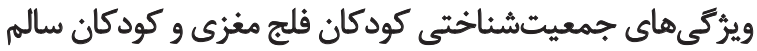

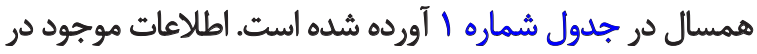

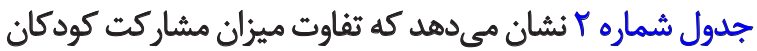

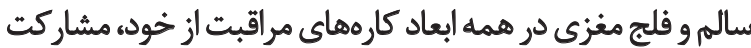

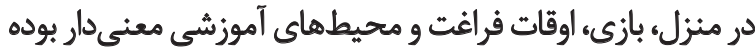

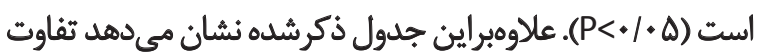

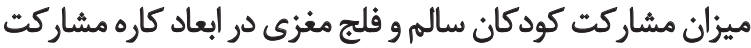

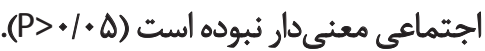

سال و تسلط والدين كودى به زبان فارسى و درك موارد يرسشنامه. دادهها از طريق نسخه فارسى يرسشنامه مشاركت كودكان (CPQ-P)

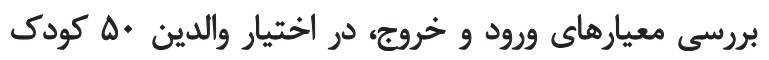

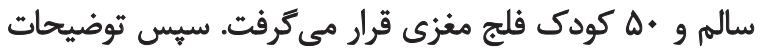

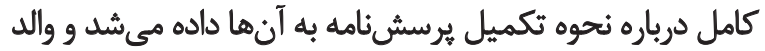

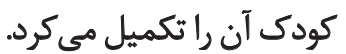

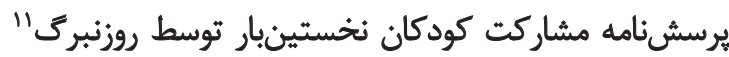

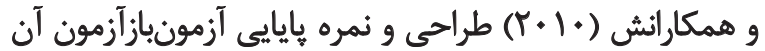

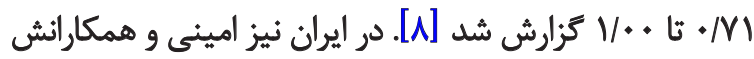

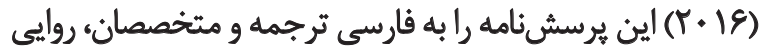

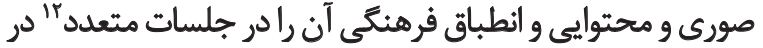

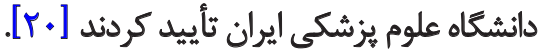

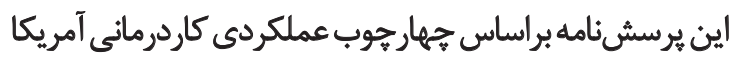

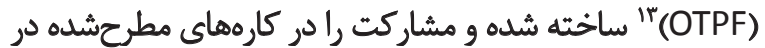

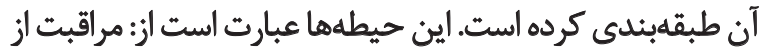

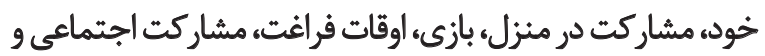

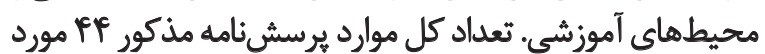

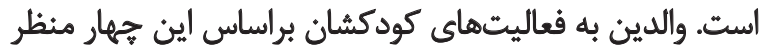

10. Children Participation Questionnaires-Persian (CPQ-P)

11. Rosenberg

12. Expert panel

13. Occupational Therapy Practice Framework (OTPF)

جدول اـ اطلاعات جمعيثشئاحتي و باليني كودكان فلج مغزى و كودكان سالم همسال \& ثاع سال.

\begin{tabular}{|c|c|c|c|}
\hline فراوانى (درصد) & & موارد & ويرُتى هاى جمعيتشناختى و بالينى \\
\hline$r q(M A \%)$ & يڤ" يسر & \multirow[b]{2}{*}{ فلج مغزى } & \multirow{5}{*}{ جنس } \\
\hline $11(\pi \% \%)$ & دختر & & \\
\hline$u(v e \cdot)$ & & \multirow{3}{*}{ ساله } & \\
\hline $1 \times(1) .1$ & 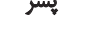 & & \\
\hline$\pi(9 \% \%)$ & دختر & & \\
\hline درصد فراوانى & فراواتى & رده سنى & 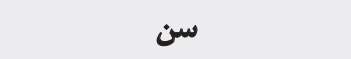 \\
\hline$r \% \%$ & 10 & allwe & \\
\hline$r+\%$ & r. & 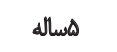 & فل فلج مثزى \\
\hline$r * \%$ & 10 & عساله & \\
\hline$\pi \%$ & ir & 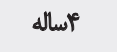 & \\
\hline$r \propto \%$ & 19 & 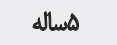 & سالم \\
\hline rA\% & 19 & توساله & \\
\hline
\end{tabular}




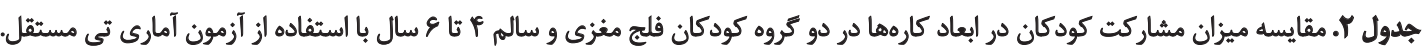

\begin{tabular}{|c|c|c|c|c|c|c|}
\hline \multirow{2}{*}{ سطح معنادارى } & \multirow{2}{*}{ ثغييرات استاندارد } & \multirow{2}{*}{ 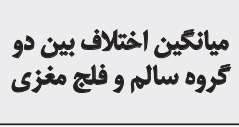 } & \multicolumn{2}{|c|}{ ميانكين نمرات } & \multirow{2}{*}{ موارد } & \multirow{2}{*}{ كار } \\
\hline & & & فلج مغزى & سالم & & \\
\hline$+1 .+1$ & $V / .1$ &.$/ \Delta T$ & $r / A$. & $\Delta / N T$ & تنوع & \\
\hline$\%$ & $\Delta / N$ & $T / M$ & $r V / F$. & $r E / T$ & شدت & \\
\hline.$/ .01$ & $9 / \Delta r$ & १/.. & $14 / 8$. & $M / \varphi$. & ميزان كمى & هراقبتبت أز خُود \\
\hline $.1 . .1$ & $q / \vee$ & $\Delta / V \lambda$ & $19 / \Delta A$ & $r A / T E$ & ميزان الذت & \\
\hline$+1+\infty$ & $9 / 97$ & $g / M$ & War & $r \Delta / A Y$ & رضايت واللدين & \\
\hline H.PT & V/Ar & . $/ \Delta P$ & $r / \Delta F$ & $r 1+1$ & تنوع & \\
\hline$\% \infty$ & $V / N$ & $T / Y \Lambda$ & $I r / g T$ & $18 / 9$. & شدت & \\
\hline $.1 .0 r$ & $9 / 48$ & PrAs & $\mid r / N T$ & IE/AT & هيزان كمى & هشاركت در مثزل \\
\hline 1.59 & $1 . / 91$ & $P / M^{e}$ & $I E M T$ & $19 / 4$ & ميزان لذت & \\
\hline$\%$ \%A & $1 . / N$ & $r / q$ & $|\Delta| \cdot A$ & IV/A & رضايت والدين & \\
\hline$\% 100 \Delta$ & $T / M A$ &.$/ 94$ & rNA & $f / r$ & تنوع & \\
\hline$\%$ & $1.18 \pi$ & $P / Q$ & ifles & $19 / 9$ & شدت & \\
\hline $.1 . .1$ & $|r / r|$ & $N Q A$ & $1 Q / M$ & $\pi / N e$ & ميزان كمى & بازى \\
\hline$* / *+1$ & $I r / M$ & s/M & $W \cdot 9$ & $\mathrm{re/aF}$ & ميزان للت & \\
\hline$+10+1$ & $\mid r / .9$ & $9 / N$ & $18 / 97$ & $\pi / \Delta$ & رضايت واللدين & \\
\hline$+1 .+1$ & $r / \pi t$ & $r / H r$ & glat & NYY & تنوع & \\
\hline $.1 . .1$ & 9/9P & V/AP & rols. & $r T / \mu$ & شيدت & \\
\hline .10 .1 & $1 \% / \uparrow A$ & $I T / M T$ & YA/FY & rNNE & هيزان كمك & اوقات فراغت \\
\hline .10 .1 & $I F / a r$ & $I E / T A$ & $\pi / N A$ & $P V / . \&$ & هيزان الثت & \\
\hline .10 .1 & $\mid Q / \cdot V$ & $1 Q / T Y$ & $r q / N$ & $\mu e / \lambda F$ & رضايت واللدين & \\
\hline.$|A+|$ & $P M$ &.$/ 1 \mathrm{~A}$ & $F / M$ & $\Delta / . \varnothing$ & تنوع & \\
\hline.$/ T T r$ & NVD & $1 / \pi$ & $I T / N$ & $\mid x / T 8$ & شدت & \\
\hline.$/ 191$ & $I T / \Delta T^{2}$ & T/AF & IV/KA & 19/9 & ميزان كمى & مشاركت اجتماعى \\
\hline.$/ T \Delta \Delta$ & $\mid r / \cdot 1$ & $r / M r$ & $m \%$ & $r E / T$ & ميزان للت & \\
\hline+1.94 & $I r / g V$ & Tr T & $r \mu / 4$ & $r \Delta / N$ & رضايت واللدين & \\
\hline$+1 *+1$ & $r / I r$ & $r / T r$ & $r / . F$ & V/RE & تنوع & \\
\hline $.1 . .1$ & $19 / \pi$ & $|V| \cdot F$ & $1 . / 9$. & tr/ar & شدت & \\
\hline $.1 . .1$ & $19 / \pi T$ & $\mathrm{H} / \mathrm{RS}$ & $1 Y / . r$ & $M / P A$ & مييزان كمى & محيطهاى آموزشى \\
\hline .10 .1 & $r T / N$ & $r \Delta / 1 P$ & 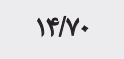 & ra/Ar & ميزان لذت & \\
\hline $.1 \cdot+1$ & $r M / T r T$ & $r \& 1 . Y$ & $1 \pi / \pi$ & $r q / T$ & رضايث واللدين & \\
\hline
\end{tabular}


مثاسب دربرابر انجام فعاليت باشد.

ميزان كمك به هنكام مشاركت در فعاليتها در بيشتر كارههاى

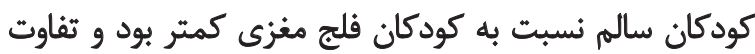

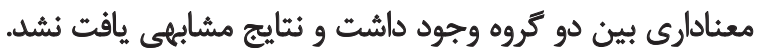

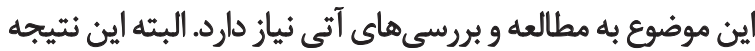

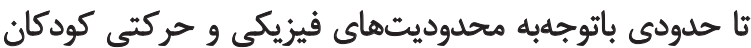

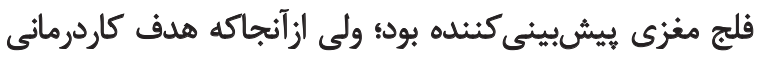

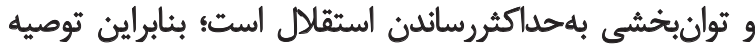

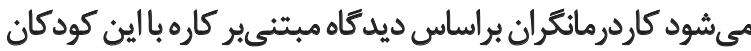

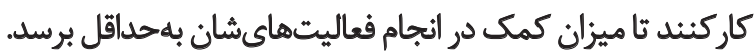

ميزان لذت از انجام فعاليتها در تمام كارههاى كودكان سالم

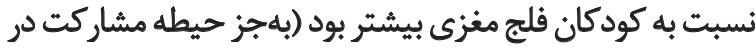

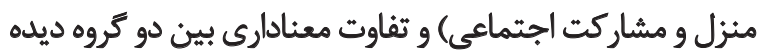

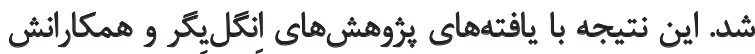

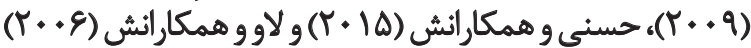

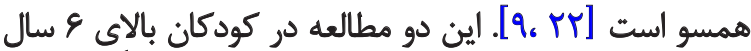

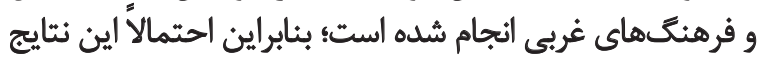

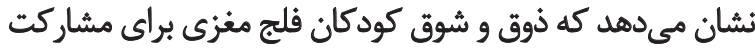

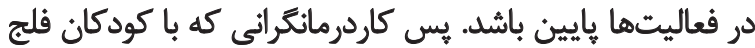

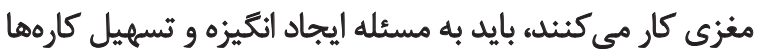

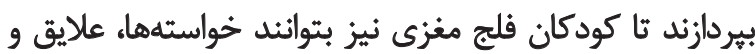

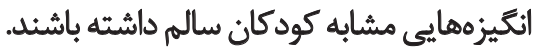

\section{نتيجهيَيرى}

نتايج نشان داد ميزان مشاركت كودكان فلج مغزى در كارهما

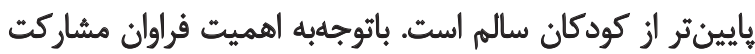

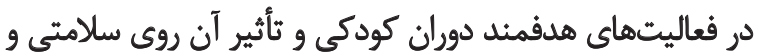

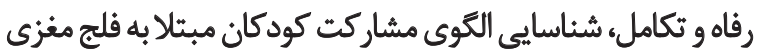

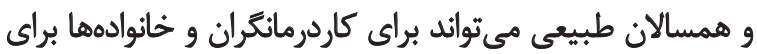

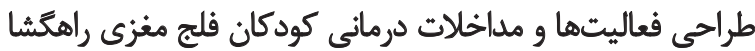

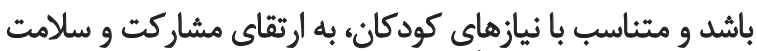

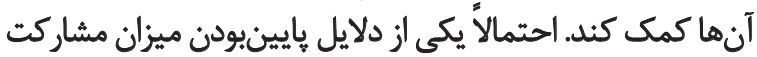

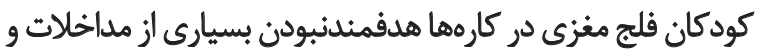

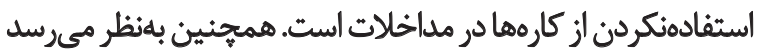

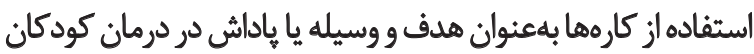

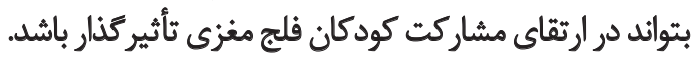

محدوديتها

محدوديتهاي موجود در مطالعه شامل محدودبودن نمونههاي

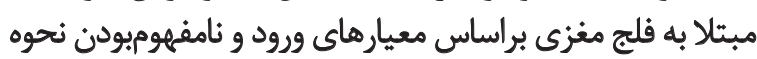

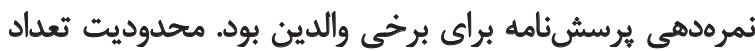

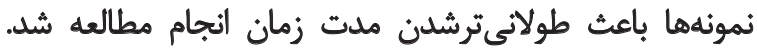

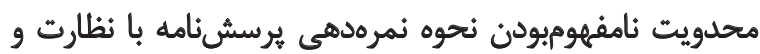

نتايج حاصل از مطالعه حاضر نشان داد كه مشاركت كودكان

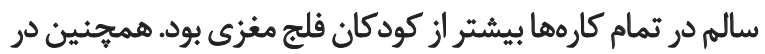

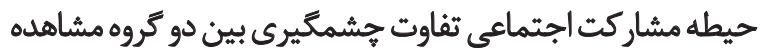

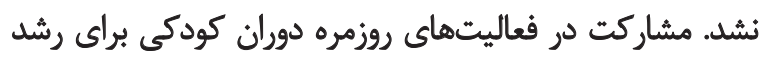

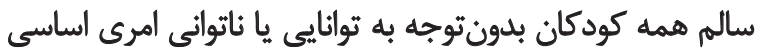

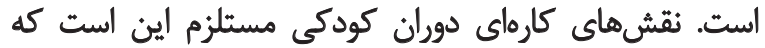

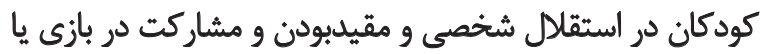

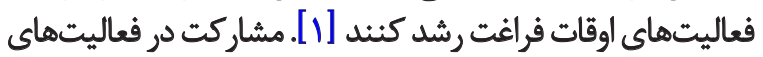

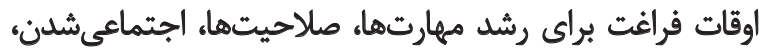

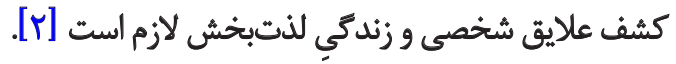
در مطالعه حاضر تنوع در مشاركت كودكان سالم همسال

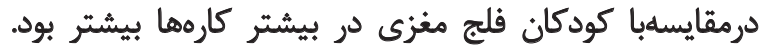

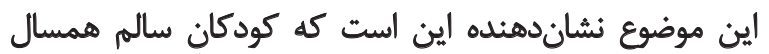

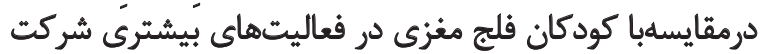

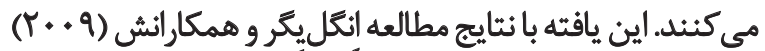

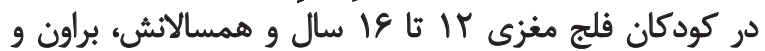

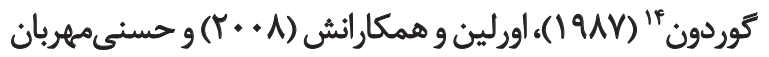

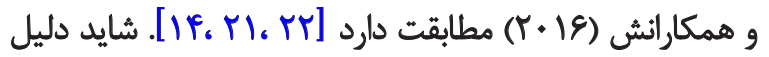

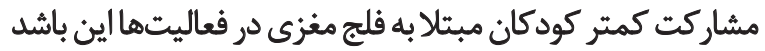

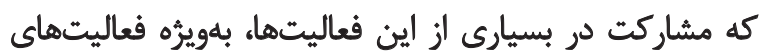

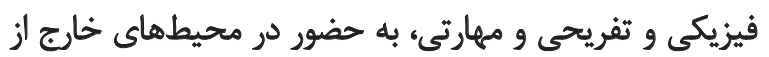

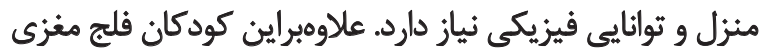

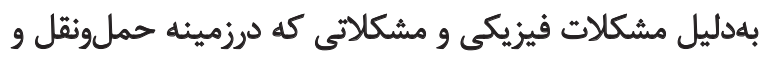

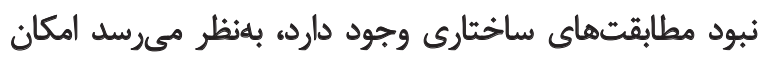
مشاركت كمترى در اين فعاليتها داشتهاند.

شايان ذكر است همه مطالعات اشارهشده، مشاركت كودكان

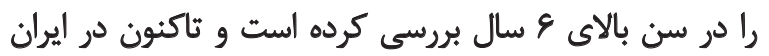

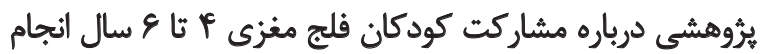

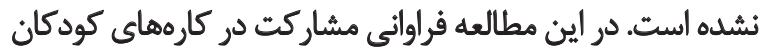

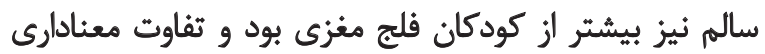

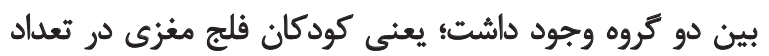

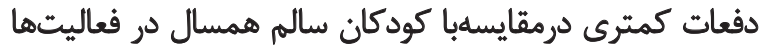

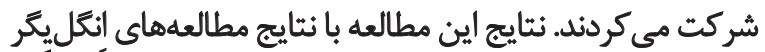

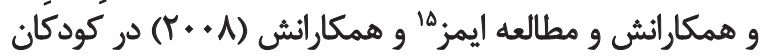

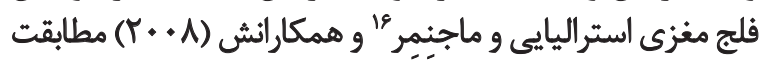

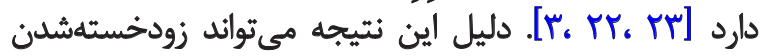

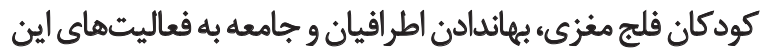

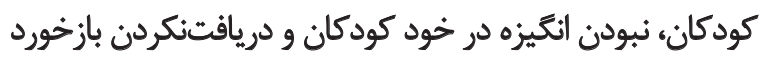

14. Brown and Gordon

15. Imms

16. Majnemer 


\section{References}

[1] Law M, Darrah J, Pollock N, Rosenbaum P, Russell D, Walter SD, et al. Focus on function-a randomized controlled trial comparing two rehabilitation interventions for young children with cerebral palsy. BMC Pediatrics. 2007; 7:31. doi: 10.1186/1471-2431-7-31

[2] Van Nieuwenhuizen O, Platenga NJ, Kasteel TE. Epilepsy in cerebral palsy: etiology, classification and prevalence. European Paediatric Neurology Society. 1997; 1(2-3):111-15.

[3] Majnemer A, Shevell M, Law M, Birnbaum R, Chilingaryan G, Rosenbaum P, et al. Participation and enjoyment of leisure activities in school-aged children with cerebral palsy. Developmental Medicine \& Child Neurology. 2008; 50(10):751-58. doi: 10.1111/j.1469-8749.2008.03068.x

[4] Soleimani F, Vameghi R, Hemmati S, Biglarian A, Sourtiii H. [Survey on types and associated disorders of cerebral palsy in Eastern and Northern Districts of Tehran (Persian)]. Archives of Rehabilitation. 2011; 12(3):75-82.

[5] Steultjens EM, Dekker J, Bouter LM, van de Nes JC, Lambregts BL, van den Ende CH. Occupational therapy for children with cerebral palsy: a systematic review. Clinical Rehabilitation. 2004; 18(1):1-14.

[6] World Health Organization. International classification of functioning, disability and health, children and youth version (ICFCY). Geneva:World Health Organization; 2007.

[7] Rosenbaum P, Paneth N, Leviton A, Goldstein M, Bax M, Damiano D, et al. A report: the definition and classification of cerebral palsy. Developmental Medicine \& Child Neurology. 2007; 109:8-14.

[8] Rosenberg L, Jarus T, Bart O. Development and initial validation of the Children Participation Questionnaire (CPQ). Disability and Rehabilitation 2010; 32(20):1633-44.

[9] Law M, King G, King S, Kertoy M, Hurley P, Rosenbaum P, Young N, Hanna S. Patterns of participation in recreational and leisure activities among children with complex physical disabilities. Developmental Medicine \& Child Neurology. 2006; 48(5):337-42.

[10] Case-Smith J. Occupational therapy for children. Use of standardized tests in pediatric practice. $5^{\text {th }}$ ed. Missouri : Elsevier. 2005.

[11] American Occupational Therapy Association. Occupational therapy practice framework: domain and process. American Occupational Therapy Association. 2014; 56:609-39.

[12] Schenker R, Coster WJ, Parush S. Neuroimpairments, activity performance, and participation in children with cerebral palsy mainstreamed in elementary schools. Developmental Medicine \& Child Neurology. 2005; 47(12):808-14.

[13] Engel-Yeger B, Jarus T, Law M. Impact of culture on children's community participation in Israel. American Journal of Occupational Therapy. 2007; 61(4):421-8.

[14] Orlin MN, Palisano RJ, Chiarello LA, Kang LJ, Polansky M, Almasri N, et al. Participation in home, extracurricular, and community activities among children and young people with cerebral palsy. Developmental Medicine \& Child Neurology. 2010; 52(2):160-6. doi: 10.1111/j.1469-8749.2009.03363.x.
توضيحات بيششتر رفع شد.

Losleing

بعد از انجام يُوهش حاضر اين يِيشنهادها مطرح مىشود:

ا. بازبيني نحوه نمرهدهي ثبرسئ نامه و تاحل ممكن ساده كردن

r. انجام اين نوع مطالعه روى كروه سنى بالاتر با استفاده از ابزار هاي ديكر؛

ا. منتقل كردن نتايج يُروهش به دانشجويان و درمانكران براي استفاده از آن در مطالعات باليني.

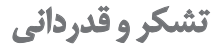

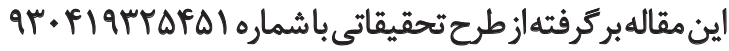

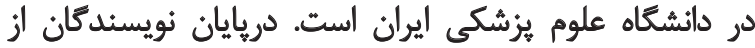

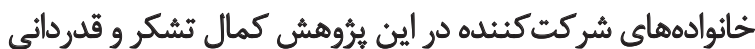
رامي كنئد. 
[15] Hassani M, Hasani Mehraban A, Aliabadi F, Taghizadeh G. [Comparison of participation between children with cerebral palsy and typically developing peers 8-14 years old in leisure activities (Persian)]. Modern Archives of Rehabilitation. 2013; 7(1):63-69.

[16] Hassani M, Hassani Mehraban A, Taghizadeh G, Aliabadi F, Ramezani S. [Enjoyment of participation in formal and informal activities among students with cerebral palsy and healthy students (Persian)]. Journal of Kermanshah University of Medical Sciences. 2015; 19(3):160-7.

[17] Nobakht Z, Rassafiani M, Rezasoltani P. Influence of child's disability on encountering environmental barriers to Participation of children with cerebral palsy. Journal of Research in Rehabilitation Science. 2013; 9(2):286-94.

[18] Nobakht Z, Rassafiani M, Rezasoltani P, Sahaf R, Yazdani F. Environmental barriers to social participation of children with cerebral palsy in Tehran. Iranian Archives of Rehabilitation. 2013; 11(2):40-45.

[19] Hassani Mehraban A, Hassani M, Amini M, The Comparison of Participation in School-Aged Cerebral Palsy Children and normal Peers: A Preliminary Study. Iranian Journal of Pediatrics. 2016. 26(3):e5303. doi: 10.5812/ijp.5303.

[20] Amini M, Hassani Mehraban A, Rostamzade O. [Translation, cultural adaptation, face, content and convergent validity of children participation into Persian (Persian)]. Archives of Rehabilitation Medicine. 2016; 5(2):151-57.

[21] Brown M , Gordon WA. Impact of impairment on activity patterns of children. Archives of Physical Medicine and Rehabilitation. 1987; 68(12):828-3.

[22] Engel-Yeger B, Jarus T, Anaby D, Law M. Differences in patterns of participation between youths with cerebral palsy and typically developing peers. The American Journal of Occupational Therapy. 2009; 63(1):96-104.

[23] Imms C , Reilly S, Carlin J, Dodd K. Diversity of participation in children with cerebral palsy. Developmental Medicine \& Child Neurology. 2008; 50(5):363-9.

[24] Soleimani F, Vameghi R, Rassafiani M, Akbar Fahimi N, Nobakht Z. Cerebral Palsy: Motor Types, Gross Motor Function and Associated Disorders. Iranian Rehabilitation Journal. 2011; 9:21-31. 